\title{
Tentative Discussion on Simplification, Unification and Ordering
}

\author{
Xuejing $\mathrm{Wu}^{1, \mathrm{a}}$ and Hongli Liang ${ }^{2, \mathrm{~b}}$ \\ ${ }^{1}$ China National Institute of Standardization, China \\ University of Chinese Academy of Sciences, China \\ awuxj@cnis.gov.cn, blianghongli@ucas.ac.cn
}

\begin{abstract}
One of the necessary conditions for a mature discipline is that its theoretical system has been established. In recent years, standardization has played an increasingly striking role in facilitating economic and trade contacts, supporting industrial development, promoting scientific and technological progress and standardizing social governance. One of the necessary conditions for the establishment of the knowledge system of the discipline of standardization is the determination of basic principle of standardization. This paper, through the review study of standardization principle both at home and abroad, finds that experts and scholars generally consider simplification, unification and ordering as the basic principle or essence of standardization. In order to distinguish them, we give the definitions of principle and method. According to the definitions we determine that ordering principle is the fundamental principle of standardization, but simplification and unification are the methods of standardization.
\end{abstract}

Keywords: Standardization, Simplification, Unification, Ordering.

\section{Introduction}

Engels said: "A nation, if wanting to be leading in the world, should always keep theoretical thinking". With historical development and social progress, standardization plays more and more striking roles. By far, standardization has become a major power for a country to develop its national competitiveness. Establishment of the knowledge system of the discipline of standardization has become very urgent for fundamentally lifting competitiveness of China's standard, establishing the discipline of standardization and training professional talents in standardization, but the basic principle of standardization has not be determined, which hinders the establishment of the knowledge system of the discipline[1-3,5]. Many experts and scholars home and abroad have carried out studies of basic principles of standardization. They unanimously think that simplification, unification and ordering are the essence or basic principle of standardization [2, 4-7].

For example, The Purpose and Principle of Standardization by British Sanders in 1972 clearly states that "standardization, in view of essence, is the practice that people consciously make it unified"; The Principle of Industrial Standardization by Japanese Matsura Shiro in 1973 expounds that "standardization is a kind of simplification in essence, and the result of conscious efforts of society"; in China, The Introduction to Foundation of Standardization by Wang Zheng in 1981 puts forward that "the unification principle of standardization is the core and essence of the principle of standardization"[3-5,8]; The Section of Standardization in the Chen Wenxiang-compiled Mechanical Industry Manual published in 1982 proposes six principles. He holds the viewpoint similar to Wang Zheng's, thinking that the unification principle is the core and essence of the principle of standardization, and the most basic principle reflecting the law of standardization activity; The Standardization System Engineering by Zhang Xichun in 1992 mentions that the ordering principle is the most fundamental principle of standardization and able to more comprehensively and deeply describe internal law of standardization activity; The Principle of Standardization by Wu Chuanzhong in 1994 advances six principle. He thinks that reasonably simplifying product size and variety is a basic principle of standardization; the article of Exploration of Basic Principle of Standardization by Liu Deju in 1997 raises that ordering principle and unification principle are basic principles of standardization, etc [2,5,8-10]. On what is the basic principle of standardization, each of experts 
sticks to his own argument. The following section will make a distinction of roles of simplification, unification and ordering principles in standardization by combining definitions of principle and method.

\section{Definitions of principle and method}

To define which of simplification, unification and ordering principles is the basic principle of standardization and which is the method for standardization, the study should begin with definitions of principle and method [1-3,5].

\subsection{Definition of principle}

According to the Word-Ocean Dictionary, "Principle" means the basic law of universal significance in a field, segment or science. Principle reflects basic law of objective things. The basic law must be of universal significance, namely only things with two characters of "universal significance" and "basic law" can be called principle. So what is law? Law means the positive connection between things and determines an inevitable trend of development of things. Law is intrinsic in thing itself, an objective reality and independent of man's will. Law and essence are closely related, and mean intrinsic fundamental attribute of things. Therefore, study of essence and study of law are of the same significance $[4,7]$. Then what is generality? Generality means abstract things existing anywhere and anytime and with common character. Any special, specific and individual thing is not of generality. For this reason, principle means the natural connection between things in development of a field, segment or science. Such natural connection exists anywhere and anytime, permeates the whole development of things from beginning to end, and influences and determines the inevitable trend of development of things. So the essence and principle of standardization means an inevitable trend of natural connection and standardization activity among things. Such natural connection and inevitable trend permeate the whole standardization activity from beginning to end, anytime, anywhere.

\subsection{Definition of method}

Method is the way, procedure and means adopted for achieving a goal, and means the skill used for completion of certain goal or task, or the procedures executed for smooth completion of things. Method means the action adopted for reaching a purpose, and every purposeful action process forms a method. In a purposeful action, people rely on a succession of actions with specific logic relations to complete some specific tasks $[5,8]$. The whole of these actions with specific logic relations is called a method. Standardization method means the scientific method to acquire survival and development advantages and establish optimal production and life orders.

\section{Analysis of simplification, unification and ordering principles}

\subsection{Analysis of simplification principle}

In terms of standardization objects with the same kind of functions, when the diversified development scale is beyond the necessary scope, the surplus, replaceable and low-function portion in the objects should be canceled, so as to guarantee them to constitute succinct, reasonable and optimal overall functions, which is the simplification principle. Simplification is a means to control chaos and disorder and to prevent diversity from spreading unchecked. It reduces number of types of things in certain scope and controls waste of kinds of standardization object due to extreme chaos and blind expansion, so as to make production and life present orderly state. According to definition of method, simplification is a means to effectively control disorder as identified by people in the practice of standardization activity. It is a specific standardization method, but not an abstract existence. Only when "its diversity development scale goes beyond necessary scope" will it be needed to carry out simplification activity. Therefore, simplification does not certainly permeate the whole standardization activity from beginning to end, and it is not of universal significance. According to analysis of definitions of principle and method, simplification principle is not the basic principle of standardization, but a standardization method [2-5]. 


\subsection{Analysis of unification principle}

In certain period and under certain conditions, unification can make form, function and other technical characters of standardization object consistent, and merge some things scattered and with characters of diversity, correlation and repeatability character scientifically and reasonably, so as to establish due order in disorder. Unification is an effective means to merge forms of expression of more than two things of the same kind into an effective means, or an effective means limited to be in a certain scope. According to definition of method, unification is similarly a means to "control disorder", with the purpose of eliminating chaos due to unnecessary diversity of things, and a standardization method to establish the order jointly complied with. The foundation of unification principle is that things to be unified must be mergeable in form, character and efficiency, namely when things are mergeable in form, character and efficiency, etc, it will be impossible to carry out unification. Therefore, unification is not of universal significance. It is not the basic principle of standardization, but a standardization method $[1-6,8]$.

\subsection{Analysis of ordering principle}

Ordering principle means the principle of carrying out human intervention in disorder with simplification, unification, generalization, seriation and combination, and limitation of all aspects of economic, technical, scientific and management activities through standard formulation, release and implementation, so as to reduce social disorder. Social material (product) and spiritual production, if not controlled, always develop to be diversified and confused, leading to worse social disorder. Standardization activity means using various standardization methods to carry out "disorder controlling" and make social disorder less. In his book Principle of Industrial Standardization, Matsura Shiro mentions that "standardization activity means our efforts to recover from disorder state to orderly state, and to object to increase of entropy in our social life." Order is the most fundamental attribute of standardization activity and reveals basic law of standardization activity.

Ordering exists in all standardization activities. Through observation, it is not hard to see that no standardization activity is not guided by ordering thought and does not target the purpose of realizing ordering finally. As the above-mentioned, simplification means reduction of number of types of things within certain scope, is a means to control chaos and disorder and prevent diversity from spreading unchecked, and helps things develop to be orderly finally from disorder through "disorder controlling". Unification is a standardization method to make things of the same object unified into one kind from more kinds or limited to certain scope, and similarly a means to "control disorder", with the purpose of eliminating chaos due to unnecessary diversity of things and establishing the order to be jointly complied with. Generalization relies on simplification of kinds and sizes of products or components and unification of structural form of products or components to enlarge the scope of use of products or components, with the purpose of preventing unnecessary diversification and making things simple and orderly. Seriation relies on reasonable planning and design of certain kind of product to make it scientifically arranged according to the optimal sequence of numbers, so as to personally control development of such products in the future and make it more orderly. Combination is an advanced form of standardization and uses composition of basic units to meet normal and reasonable demands for diversity, so as to avoid the trend of irregular diversity. Therefore, it is also a measure to lift orderliness. Ordering permeates standardization activity from beginning to end. No purpose of formulating, releasing and implementing standard is not for realization of ordering.

To sum up, ordering embodies fundamental attribute of standardization activity, and reveals basic law of standardization activity. In addition, ordering permeates the whole standardization activity from beginning to end, and guides conduction of standardization activity all the time. Analysis of definition of principle shows that ordering principle is the basic law of universal significance for standardization activity. Therefore, ordering principle is the basic principle of standardization. 


\section{Conclusion}

Review and study of domestic and foreign principles of standardization get the following findings: experts unanimously think that simplification principle, unification principle and ordering principle are basic principles of standardization. This paper carries out analysis of the three principles according to definitions of principle and method, and finally thinks that simplification and unification are methods for standardization, and ordering principle is the basic principle of standardization activity. Determination of the basic principle for standardization will move ahead with establishment of the knowledge system of standard science and development of the discipline of standard science.

\section{Acknowledgements}

This research was financially supported by the Special Project of Quality Inspection of Public Welfare Industry Research "Study the Methods to Choose and Determine the Key Elements for Technical Standards(No.: 201310225)", "Research on the Classification Analyzation and Rule of Development for Standards Based on the Technical Contents(No.: 201210208)"; Project of Standardization Administration of the People's Republic of China "Research on Transformation Mechanisms for International Standards and Advanced Foreign Standards (European Union)"(No.: 572014B-3395); "Research on Construction of Recommended Standard Systems" (No.: 572014B-3590); "Research on Construction of Compulsory Standard Systems" (No.: 572013B-3098).

\section{References}

[1] Wang, Z.: On Methods for Standardization [J]. Study and Exploration. 1999 (1).

[2] Zhao, W., Bai, D., Zhao, C.: Tentative Discussion on Naming of the Discipline of "Standard Science" and the Studies [J]. Standard Science. 2009(7).

[3] Liu, D.: Exploration of Basic Principle of Standardization [J], Aerospace Standardization, 1997(1).

[4] Li, C.: General Introduction to Standardization [M]. Beijing: China Renmin University Press.

[5] Zhang, X.: Standardization System Engineering [M]. Beijing: Beihang University Press.

[6] [UK] T.R.B. Sanders: Purpose and Principle of Standardization [M]. Beijing: Scientific and Technical Documents Publishing House.

[7] [Japan] Matsura Shiro: Principle of Industrial Standardization [M]. Beijing: Technical Standard Publishing House.

[8] Wang, Z.: The Introduction to Foundation of Standardization[M]. Beijing: Technical Standard Publishing House.

[9] Research Institute of Science, Ministry of Machine-Building Industry, Mechanical Industry Manual [M]. Beijing: Chemical Industry Press.

[10] Wu, C.: Principle of Standardization [M]. Guizhou: Guizhou Administration of Standard and Measurement. 